\title{
The differential effects of unihemispheric and bihemispheric tDCS over the inferior frontal gyrus on proactive control
}

\author{
Jorge Leite ${ }^{a, b, c, *}$, Óscar F. Gonçalves ${ }^{a, c}$, Patrícia Pereira ${ }^{a}$, Niranjan Khadka ${ }^{d}$, \\ Marom Bikson ${ }^{d}$, Felipe Fregni ${ }^{c}$, Sandra Carvalho ${ }^{\mathrm{a}, \mathrm{c}}$ \\ a Neuropsychophysiology Laboratory, CIPsi, School of Psychology (EPsi), University of Minho, Campus de Gualtar, 4710-057 Braga, Portugal \\ b Univ Portucalense, Portucalense Institute for Human Development - INPP, Oporto, Portugal \\ c Spaulding Neuromodulation Center, Department of Physical Medicine and Rehabilitation, Spaulding Rehabilitation Hospital and Massachusetts General \\ Hospital, Harvard Medical School, Boston, MA, USA \\ ${ }^{\mathrm{d}}$ Department of Biomedical Engineering, The City College of New York of CUNY, New York, NY, 10031 USA
}

\section{A R T I C L E I N F O}

\section{Article history:}

Received 16 February 2017

Received in revised form 14 August 2017

Accepted 18 August 2017

Available online 23 August 2017

\section{Keywords:}

Prepotent behavior

Proactive control

Unihemispheric tDCS

Bihemispheric tDCS

Right inferior frontal gyrus

\begin{abstract}
A B S T R A C T
This study examined the effects of bihemispheric and unihemispheric transcranial Direct Current Stimulation (tDCS) over the inferior frontal gyrus (IFG) on proactive control.

Sixteen participants were randomized to receive (i) bihemispheric tDCS, with a $35 \mathrm{~cm}^{2}$ anodal electrode of the right IFG and a $35 \mathrm{~cm}^{2}$ cathode electrode of left IFG or (ii) unihemispheric tDCS, with a $35 \mathrm{~cm}^{2}$ anodal electrode of the right IFG and a $100 \mathrm{~cm}^{2}$ electrode of the left IFG or (iii) sham tDCS, while performing a prepotent inhibition task. There were significant speed-accuracy tradeoff effects in terms of switch costs: unihemispheric tDCS significantly decreased the accuracy when compared to bihemispheric, and sham tDCS, while increased response time when comparing to bihemispheric and sham tDCS. The computational model showed a symmetrical field intensity for the bihemispheric tDCS montage, and an asymmetrical for the unihemispheric tDCS montage. This study confirms that unihemispheric tDCS over the rIFG has a significant impact on response inhibition. The lack of results of bihemispheric tDCS brings two important findings for this study: (i) left IFG seems to be also critically associated with inhibitory response control, and (ii) these results highlight the importance of considering the dual effects of tDCS when choosing the electrode montage.
\end{abstract}

(c) 2017 Elsevier Ireland Ltd and Japan Neuroscience Society. All rights reserved.

\section{Introduction}

The ability to inhibit response tendencies or stopping an ongoing response is a key element to understand adaptive goal direct behavior (Bari and Robbins, 2013). This ability is also crucial for cognitive flexibility, because in order to successfully change from one rule to another, one of the rules needs to be successfully inhibited. For instance, the role of the right inferior frontal gyrus (rIFG) and deficits in proactive control (i.e., anticipation of stopping based on contextual cues) has already been reported (van Rooij et al., 2014). Several neuroimaging studies have supported this association by suggesting that the right inferior frontal gyrus (IFG) has a key role on the mediation of response inhibition (Aron et al., 2004; Garavan

\footnotetext{
* Corresponding author at: Spaulding Neuromodulation Center, Department of Physical Medicine and Rehabilitation, Spaulding Rehabilitation Hospital, Harvard Medical School, 96 13th Street, Charlestown, 02129, Boston, MA, USA.

E-mail address: jleite@neuromodulationlab.org (J. Leite).
}

et al., 2006; Garavan et al., 1999; Kelly et al., 2004). Furthermore, studies using anodal transcranial direct current stimulation (tDCS) to the right IFG were able to increase response inhibition in stop signal tasks (Cunillera et al., 2014; Ditye et al., 2012; Hogeveen et al., 2016; Jacobson et al., 2011; Stramaccia et al., 2015). However, it is still unclear whether IFG control on proactive control involved in response inhibition is truly a lateralized function (i.e., left IFG would have a minimal impact) or if it depends on the successful mediation between right and left IFG. One possibility to study this is by testing unihemispheric versus bihemispheric tDCS.

tDCS is a non-invasive method of brain stimulation that is able to induce polarity specific changes in the neural membrane potential and excitability (Nitsche and Paulus, 2000, 2001). Consequently, tDCS can be used as a tool to study the effects of increased neural engagement of a given circuit activated by a cognitive task. Computer modeling studies predict the resulting brain current flow for a given electrode placement (montage); and while the relationship between tDCS montage and resulting brain current flow is not trivial, it can be addressed with modeling (Peterchev et al., 
2012). A concern in tDCS research is that its effects are not due to one electrode only but by the combination of effects between two electrodes (anode and cathode polarity) with potentially opposite characteristics. This has been the case with most of the studies exploring the effects of tDCS on response inhibition (Stramaccia et al., 2015).

Some studies have attempted to leverage the actions of both electrodes synergistically. Studies using bihemispheric ("lateralized") montages are based on the assumption that by stimulating simultaneous homotopic regions (with an anode and a cathode), the inter-hemispheric balance will shift towards the anode, thus potentially favoring the cognitive processing performed on that hemisphere (the one receiving anodal tDCS) (Jacobson et al., 2012). Several studies targeting craving reduction have used bihemispheric tDCS montages successfully (Batista et al., 2015; Wietschorke et al., 2016; Yavari et al., 2016), a cognitive process that involves inhibitory control. But surprisingly there are no effects of these bihemispheric montages on response inhibition in healthy participants (Dambacher et al., 2015).

The evidence so far seems to suggest that the rIFG may be the main involved in response inhibition. Given this evidence, we hypothesized that anodal tDCS of rIFG would enhance response inhibition. We further wanted to test whether decreasing excitability in the left IFG would have a synergistic effect. Therefore, in this study we addressed this question by placing a $35 \mathrm{~cm}^{2}$ electrode over the right IFG, and using two electrode sizes over the left IFG, namely $35 \mathrm{~cm}^{2}$ (for bihemispheric tDCS) and $100 \mathrm{~cm}^{2}$ (for un-ihemispheric tDCS). The rationale behind these two types of montages is that with similar electrode sizes, the electrode current density between them identical producing neuromodulation (though with opposite polarity) under both electrodes. But when one electrode is larger than the other one, the current density will be smaller on the larger electrode, producing neuromodulation only under the smaller (Nitsche et al., 2007). This rationale was addressed with computational modeling. Behaviorally we then tested if the processing over the rIFG is segregated, increased activity of that region, with or without decreased left IFG activity will benefit right hemisphere processing, and thus will increase task performance.

\section{Methods}

\subsection{Participants}

A total of sixteen-college student volunteers (age: $21.5 \pm 4.5,11$ females) naïve to tDCS participated in this study. All participants were right-handed (Edinburgh Handedness Inventory: $\mathrm{EHI} \geq 80$ ), healthy, with normal or corrected-to-normal visual acuity and without present or past history of neurological or psychiatric disorders. Participants were excluded if in the 4 weeks prior to the study they were using any medication or psychotropic drugs. Participants were advised to avoid alcohol, cigarettes and caffeinated drinks on the day of the experiment, and none reported fatigue due to insufficient sleep. All participants gave their written informed consent prior to their inclusion in the study and the study was performed in accordance with the Declaration of Helsinki.

\subsection{Design}

This experiment consisted of 3 sessions, with an intersession interval of at least $72 \mathrm{~h}$. The experimental design of each session comprised 3 moments: 1) Pre-task assessment; 2) Prepotent Inhibition Task+tDCS; and 3) Post-task assessment. In the pre and post-task assessments participants were screened about possible levels of discomfort, fatigue, pain, itching, humor, tingling, burning, headache and sleepiness (among others) using a Visual Analogue Scale (VAS). The objective of this assessment was to evaluate possible secondary effects due to the stimulation. Participants performed the tasks after 3 min of tDCS. Task order was kept constant in order to minimize potential differences due to stimulation timing.

\subsection{Main task}

\subsubsection{Prepotent response inhibition task}

This task is a modification of the Preparing to Overcome Prepotency task (Snitz et al., 2005). In this task participants were instructed to attend to a cue (a green or a red square) and then to respond to a target presented in the center of the screen (an arrow pointing to the left or to the right; or upward and downward). If the cue presented on the screen was green, participants should respond left or right following the direction of the target using the left or right index respectively. If the cue color was red, participants should instead respond in the opposite direction of the target presented on screen. This prepotent response inhibition task implies a cue-dependent rule change. However, instead of requiring a response stop, it requires that the participant performs an alternate response. The full experiment consisted of 120 trials, with 84 prepotent response trials (i.e., answer left and right to arrows pointing to left and right, respectively), and 36 trials prepotent response inhibition trials (i.e., answer left and right to arrows pointing to right and left, respectively). The cues were presented for 500-msec and targets were presented for 3000-msec, with a jittered intertrial interval varying between 1000, 1500, and 3000-msec. Before each cue, a fixation cross in the center of the screen was presented for 500-msec, directing participantís attention to the center of the screen (see Fig. 1).

\subsection{Control tasks}

\subsubsection{Choice Reaction Time (CRT)}

In this task a target was presented in the center of the computer screen requiring participants to respond as fast as possible. There were a total of 64 trials. For each trial, a fixation cross appeared at the center of the computer screen for $200,400,600$, or $800 \mathrm{msec}$ before each target. Participants were required to press the " $\mathrm{X}$ " key with their left index finger if a "*” appeared on the screen, and to press "N" key with the right index finger if "\#" appeared on the screen. There was a $50 \%$ probability for each target to be presented on the screen. The duration of this task was approximately $2 \mathrm{~min}$.

\subsubsection{Go No-Go task}

In this task, stimuli were presented in a continuous stream and participants were asked to press the "space" key on a computer keyboard when a letter was presented in the center of the computer screen (go trials), and withhold the response only when the letter "X" was presented (no go trials). The task consisted of a total of 148 trials, 25 no go trials and 123 go trials. Each stimulus was presented for 500-msec, followed by a fixation cross with a duration of 1000 msec.

\subsection{Transcranial direct current stimulation (tDCS)}

tDCS was applied using $35 / 35 \mathrm{~cm}^{2}$ (bihemispheric tDCS) and $35 / 100 \mathrm{~cm}^{2}$ (uni hemispheric tDCS) saline-soaked electrode sponges, using an Eldith DC Stimulator Plus (Neuroconn, Germany). The experiment had a within-subject design, in which all participants were randomized to receive 3 tDCS conditions in a counterbalanced manner: 2 active (bihemispheric tDCS and unihemispheric tDCS) and one sham (with uni- or bihemispheric tDCS montage). The anode electrode was placed over the right inferior 


\section{Prepotent Inhibition Task}

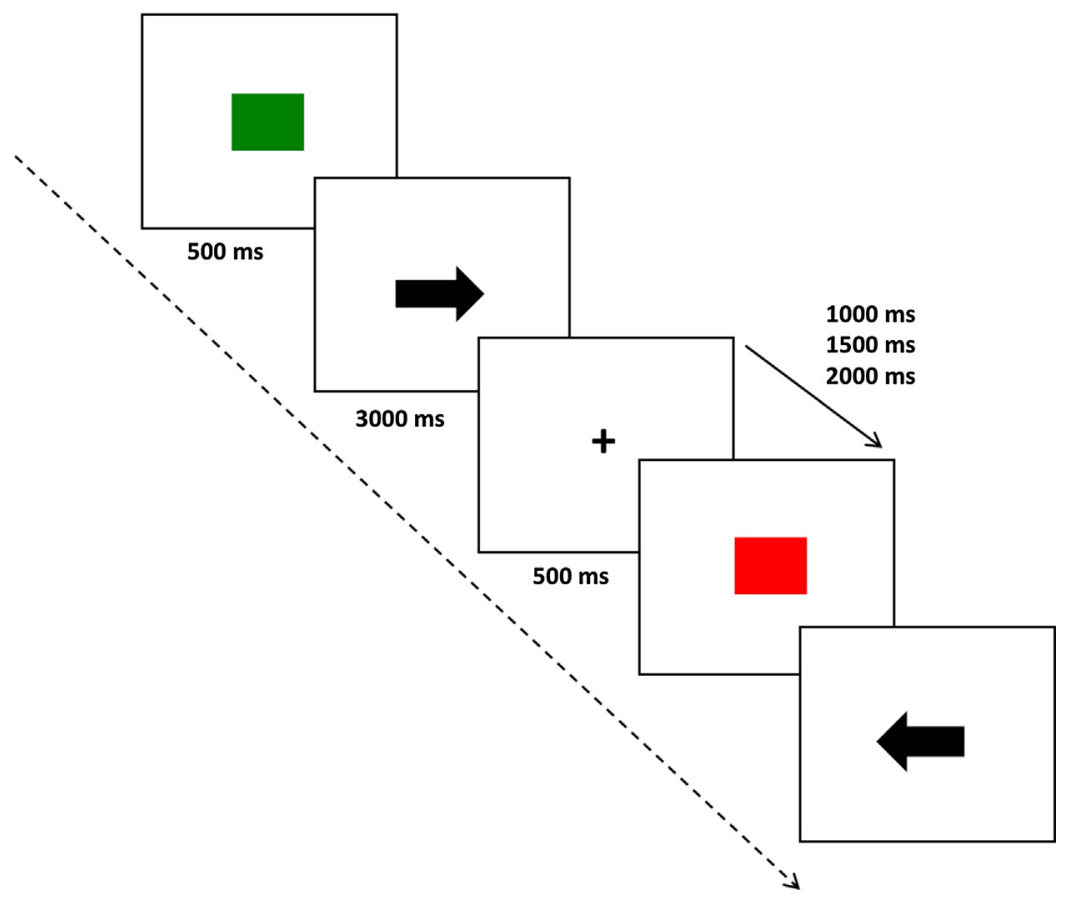

Fig. 1. Schematic representation of the Prepotent Inhibition Task.

frontal gyrus (rIFG) and the cathode over the left inferior frontal gyrus (IIFG), using two rubber bands. There was at least $6 \mathrm{~cm}$ distance between the anode and the cathode. The IFG placement was determined based on the international 10-20 system as previously performed by Kim and colleagues (Kim et al., 2007). A 1 mA intensity DC was applied for about $30 \mathrm{~min}$, during the entire duration of the task (with a 15-s ramp up and down) for the active condition, while for sham the duration was 15-s (with a 15-s ramp up and down).

\subsection{Computational model}

An exemplary magnetic resonance imaging (MRI) scan of a template head was segmented by ScanIP software (Simpleware, Exeter, UK) into seven tissue masks namely scalp, fat, skull, CSF, gray matter, white matter, and air to develop a high resolution $(1 \mathrm{~mm})$ MRI derived finite element method (FEM) model. Conductivity value for each tissue mask was based on prior literature (Bikson et al., 2015). Electrodes for uni-hemisphere montage $\left(35 / 100 \mathrm{~cm}^{2}\right.$ scalp contact area) and bi-hemisphere montage $\left(35 / 35 \mathrm{~cm}^{2}\right.$ scalp contact area) positioned over IFG (Fig. 5A1 and B1) were first imported into the head model, and the resulting volumetric meshes were later imported into COMSOL Multiphysics 4.3 (COMSOL Inc., MA, USA) to solve the model. The final FEM head assembly was solved for greater than $10,000,000^{\circ}$ of freedom and had greater than $12,000,000$ tetrahedral elements. For electrical stimulation, a quasistatic approximation was implemented and the boundary conditions were applied as normal current density (inward current flow: Jnorm) at the top exposed surface of anode $(1 \mathrm{~mA})$ and ground at the top surface of return electrode. Other remaining external surfaces of the model were electrically insulated. Electric field streamlines (seeded uniformly from the top surface of electrode and proportional to the logarithm of field intensity magnitude) were generated for each montage (A2, B2) to illustrate the distribution of field across different head tissues.

\subsection{Data analysis}

Switch costs index for Response time (RT) were calculatedsubtracting the mean RT of responses to prepotent cue responses from the mean RT of responses to non-prepotent ones. Switch costs for accuracy were calculated subtracting the accuracy from responses to prepotent cues from responses to non-prepotent ones. Therefore, higher values suggest worse performance, while values close to zero or negative suggest better performance.

We performed two one-way repeated measures ANOVAs with three levels (unihemispheric, bihemispheric, and sham tDCS) for switch cost accuracy and response time. We also performed two way repeated measures ANOVAs with condition (prepotent, non prepotent) and tDCS (unihemispheric, sham and bihemispheric tDCS) as factors for analyzing participants task performance.

In order to detect any discriminability differences during task performance we performed another one-way repeated measures ANOVA for dí (d-prime or signal detection). Dí was calculated by using the hit ratio from the nonprepotent condition, and the error ratio from the prepotent condition, as the later would be equivalent to a false positive for the non-prepotent condition, using the following formula:

$d^{\prime}=z$ (Hits $)-z$ (FalseAlarms $)$

Moreover, all the control tasks were analyzed by the means of one-way repeated measures ANOVAs.

\section{Results}

\section{1. tDCS self-report side effects}

No moderate or severe side effects were reported after tDCS. 


\subsection{Prepotent inhibition task}

\subsubsection{Task analysis}

There were no significant effects of tDCS $\left[\mathrm{F}(2,30)=1.661, \mathrm{p}=0.217, \eta_{\mathrm{p}}{ }^{2}=0.100, \varepsilon=0.547\right]$, nor significant interaction between tDCS and trial condition $[F(2,30)=0.148, p=0.863$, $\left.\eta_{\mathrm{p}}{ }^{2}=0.010\right]$ for accuracy. But, as expected, there was a main effect of trial condition $\left[F(1,15)=7.003, p=0.018, \eta_{p}{ }^{2}=0.318\right]$, in which participants responded significantly with more accuracy to prepotent trials $(\mathrm{M}=96.578, \mathrm{SE}=1.233)$ than to non-prepotent ones $(\mathrm{M}=94.154, \mathrm{SE}=1.471)(\mathrm{p}=0.018)$ (Fig. 2).

Similarly, for response time, there were no significant effects of $\operatorname{tDCS}\left[\mathrm{F}(2,30)=0.549, \mathrm{p}=0.583, \eta_{\mathrm{p}}{ }^{2}=0.035\right.$, ], nor significant interaction between tDCS and trial condition $[\mathrm{F}(2.30)=1.453, \mathrm{p}=0.250$, $\left.\eta_{\mathrm{p}}{ }^{2}=0.088\right]$. But, as expected, there was a main effect of trial condition $\left[\mathrm{F}(1,15)=6.255, \mathrm{p}=0.024, \eta_{\mathrm{p}}^{2}=0.294\right]$, in which participants responded significantly faster to prepotent trials $(M=629.342$, $\mathrm{SE}=30.010)$ than to non prepotent ones $(\mathrm{M}=657.580, \mathrm{SE}=32.488)$ $(\mathrm{p}=0.024)$ (Fig. 2).

\subsubsection{Switch cost accuracy}

There were significant effects in terms of switch costs accuracy $\left[F(2,30)=5.284, p=0.007, \eta_{p}^{2}=0.280\right]$. Post hoc pairwise LSD comparisons showed that unihemispheric tDCS $(\mathrm{M}=0.754, \mathrm{SE}=0.827)$ significantly decreased the switch cost when compared to both bihemispheric tDCS $(M=2.779, S E=0.742)(p=0.002)$ and sham $\operatorname{tDCS}(\mathrm{M}=1.885, \mathrm{SE}=0.903)(\mathrm{p}=0.035)$. Additional analysis revealed that this effect was not secondary to a change in response criterion $\left[\mathrm{F}(2,30)=1.391, \mathrm{p}=0.263, \eta_{\mathrm{p}}^{2}=0.085, \varepsilon=0.660\right]$ (Fig. 3).

\subsubsection{Switch cost performance (response time)}

There were significant effects in terms of switch cost for response times $\left[\mathrm{F}(2,30)=4.737, \mathrm{p}=0.016, \eta_{\mathrm{p}}{ }^{2}=0.240\right]$. Post hoc pairwise LSD comparisons showed that unihemispheric tDCS $(M=42.278, S E=7.024)$ significantly increased the switch cost when comparing to both dual hemispheric $(\mathrm{M}=6.509, \mathrm{SE}=13.538)$ $(\mathrm{p}=0.015)$ and sham tDCS $(\mathrm{M}=20.212, \mathrm{SE}=10.220)(\mathrm{p}=0.042)($ see Fig. 3).

\subsection{Control tasks}

There were no significant effects of tDCS on Choice Reaction Time $\left[F(2,30)=1.452, \mathrm{p}=0.250, \eta_{\mathrm{p}}{ }^{2}=0.088\right]$, nor on proactive inhibition $\left[\mathrm{F}(2.30)=1.170, \mathrm{p}=0.324, \eta_{\mathrm{p}}^{2}=0.072\right]$, as measured by the response time in the go trials from the go/no go task. There were also no difference in terms of accuracy for the no go trials $\left[\mathrm{F}(2.28)=0.095, \mathrm{p}=0.837, \eta_{\mathrm{p}}{ }^{2}=0.007, \varepsilon=0.686\right]$ (Fig. 4).

\subsection{Computational model predicted field}

The computational model predicted field intensity (Fig. 5 A3 and B3) and trajectories of current flow (Fig. 5A2 and B2) through gray matter. The results from these FEM simulations were broadly consistent with the montage design intentions. A symmetrical field intensity was predicted for the dual-hemisphere tDCS montage (Fig. 5A3), whereas, field was asymmetrical (smaller field intensity/current density under large electrode compared to the small electrode) for the uni-hemisphere tDCS montage (Fig. 5B3).

\section{Discussion}

This study tested the effects of unihemispheric, bihemispheric, and sham tDCS over the right inferior frontal gyrus on inhibitory control, as assessed by the Prepotent Response Inhibition Task (PRIT). We hypothesized that if prepotent response inhibition is mostly associated with rIFG control then by decreasing excitability in the left IFG with tDCS (bihemispheric condition), inhibition would be larger.

Our results showed that unihemispheric tDCS increased accuracy, but at the cost of response speed when comparing to bihemispheric and sham tDCS in the PRIT. This task involves a response (left or right) to a cue pointing towards the same direction that subjects should respond. In approximately $20 \%$ of the trials, participants should inhibit the prepotent response elicited by the arrow cue, and respond to the opposite direction (nonprepotent response). Therefore, the successful completion of the task is dependent on the proactive inhibition of the non-prepotent response upon the cue presentation.

Only unihemispheric tDCS induced slowing of motor process in the presence of non-prepotent stimuli (Aron et al., 2014) - which is thought to be a function of the rIFG. These effects of tDCS on response time do not seem to be due to an overall slowing process of responses, as shown by the lack of effects on the choice reaction time task.

SAT is a dynamic and inverse process that is often observed between accuracy and speed when performing a task. That is, increased response times can occur with a cost of reduced accuracy; or response speed is decreased in order to increase overall accuracy (Bogacz et al., 2010). Despite several theories hypothesizing on how the SAT occurs, what is well known is that it is dependent on a cortico-basal ganglia-thalamic circuit (Bogacz et al., 2010). For instance, in a scenario with accuracy emphasis, the subthalamic nucleus receives increased input from frontal regions (Frank et al., 2007). Thus it is possible that unihemispheric tDCS was able to increase these outputs to subcortical regions, which in turn led to slower, but more accurate responses. These tDCS effects on accuracy but not response time have already been showed in the past (Bolognini et al., 2010; Carvalho et al., 2015; Fregni et al., 2005; Zaehle et al., 2011). Accuracy increase has been thought to represent a top down control mechanism. For instance, high performers in a paced tapping task relied more on top down control of motor and sensory regions and low performers on a bottom up approach (Witt and Stevens, 2013). Thus, polarity induced effects of tDCS may impact cognition/behavior through a top down modulation instead of a bottom up (Morales-Quezada et al., 2016). Moreover, a study by Leite et al. (2013) using bihemispheric tDCS over the DLPFC showed that on complex versions of a task switching task, increased accuracy was associated with decreased response speed. Not surprisingly, the IFG is one of the areas involved in the cortico-subcortical network that is responsible for the SAT (Bogacz et al., 2010). It is possible that increased activity over the rIFG also increased the activity over the subthalamic nucleous (STN), that subsequently led to slower, yet more accurate, responses (Aron et al., 2007; Aron and Poldrack, 2006).

Given the results with unihemispheric tDCS, we would expect that by simultaneously decreasing excitability in the left IFG would make the effects larger or at least of the same magnitude. However, the results for bihemispheric tDCS had the opposite results to what we a priori predicted: when excitability diminishing cathodal tDCS of left IFG was combined with anodal tDCS to the rIFG, the results of anodal rIFG tDCS disappeared. These results suggest that left IFG is also critically associated with inhibitory cortical response. This is supported by evidence from human lesion studies, in which the left IFG was associated with impairments in inhibitory control during the performance of a go-no go task (Swick et al., 2008). Moreover, Hampshire et al. (2010) showed that there was a bilateral activation over the IFG during a stop signal task. Moreover, no data to support the uniqueness or specialized role of the rIFG during response inhibition was found. And interestingly enough, an EEG study also suggested the functional relevance of left sided regions for the successful response inhibition and switching, which 
A1

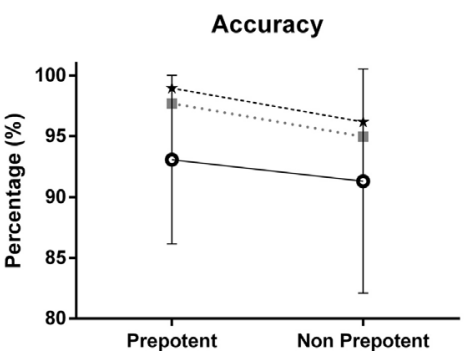

A2

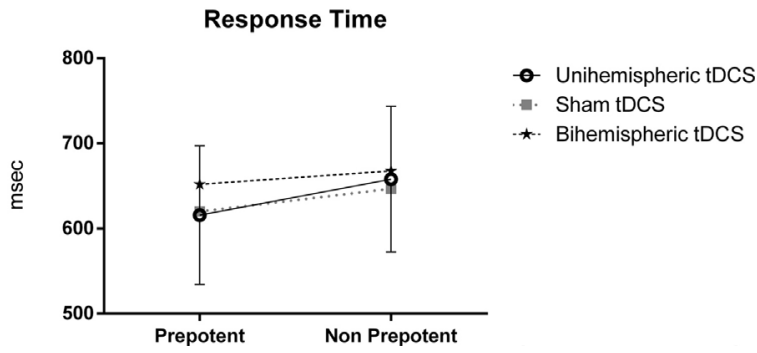

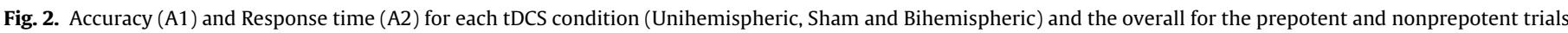
(P value $<0.05)$.

A1

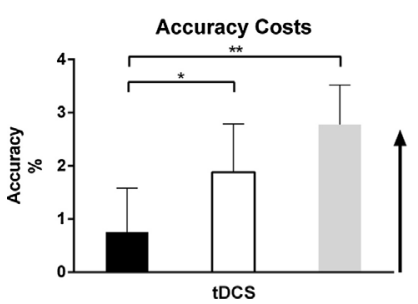

A2

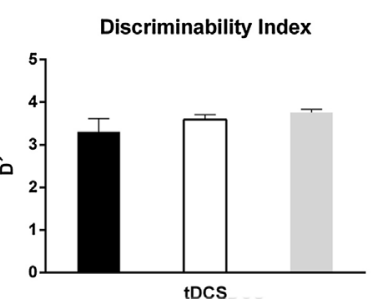

A3

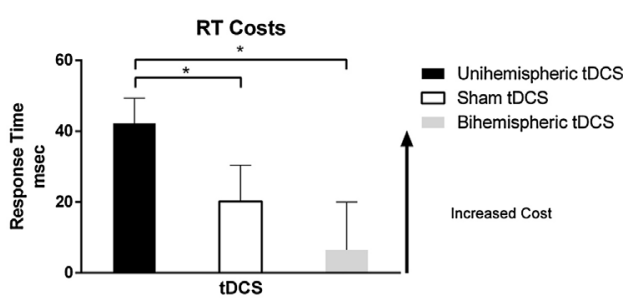

Fig. 3. Switch costs accuracy (A1), discriminability index (A2), and response time (RT) costs (A3) ( ${ }^{*} \mathrm{P}$ value $<0.001$, ${ }^{* *} \mathrm{P}$ value $\left.<0.05\right)$.
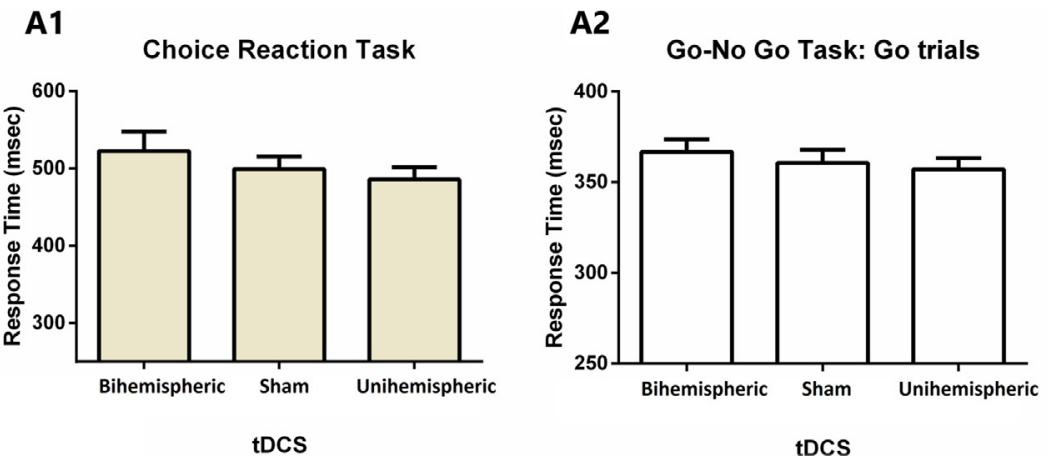

A3

Go-No Go Task: No Go trials

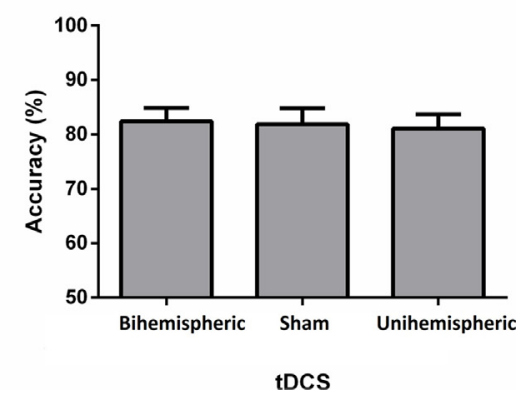

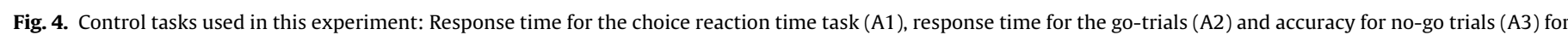
the go-no go task.

may suggest the role of the left hemisphere in action selection (Serrien et al., 2013). Our results also underscore the role of left IFG in response control. Left IFG has indeed been suggested to be involved in switching between stimulus categorizations (Philipp et al., 2013), which may be also recruited during the performance of the PRIT. One could argue that this deleterious effect of bihemispheric tDCS on task performance was due to decreased activity over the left IFG induced by the cathode. Although possible, a previous study using tDCS over the left DLPFC did not find any significant differences in switching between stimulus categorization after cathodal tDCS (Leite et al., 2011). Thus, it is also possible that this deleterious effect of bihemispheric tDCS cannot be explained by the influence of the cathode per se over the left IFG, but also due to differences of current distribution between uni vs. bihemispheric, as shown by the computer modeling (Fig. 5).

Nonetheless, the specific role of the IFG on response inhibition has been challenged recently. Using several fMRI tasks, Erika-Florence et al. (2014) did not find any specific activations in the IFG related to response inhibition, but instead suggested that response inhibition relies on several spatially distributed functional fronto-parietal networks related to specific cognitive demands (e.g., Erika-Florence et al., 2014). Additionally, a study comparing homotopic recruitment in young and elder subjects, showed that elder people recruited additional brain areas in order to meet task demands (Davis et al., 2012). Our study also highlighted that functional changes in the brain (induced for instance by aging) will be dependent on the existing neural structure, which may impact the recruitment within the brain network. Such changes are not only dependent on aging, but from a variety of factors, tDCS included. For instance, tDCS has already been shown to change resting state functional connectivity (Kunze et al., 2016; Mondino et al., 2016). Therefore, it may be possible that the combination of single or bihemispheric tDCS with task performance, induced very distinct network activations. This is certainly a possibility that could help explaining the present results, as on a previous study the polarity induced effects of bihemispheric tDCS over the dorsolateral PFC showed an interaction effect with the level of task difficulty, thus producing distinct behavioral effects (Leite et al., 2013). One potential limitation of the present study is that it cannot fully test the collaborative hypothesis between homotopic regions- as it is possible that anodal tDCS delivered bilaterally to the homotopic IFG can increase prepotent response inhibition. Future studies should com- 

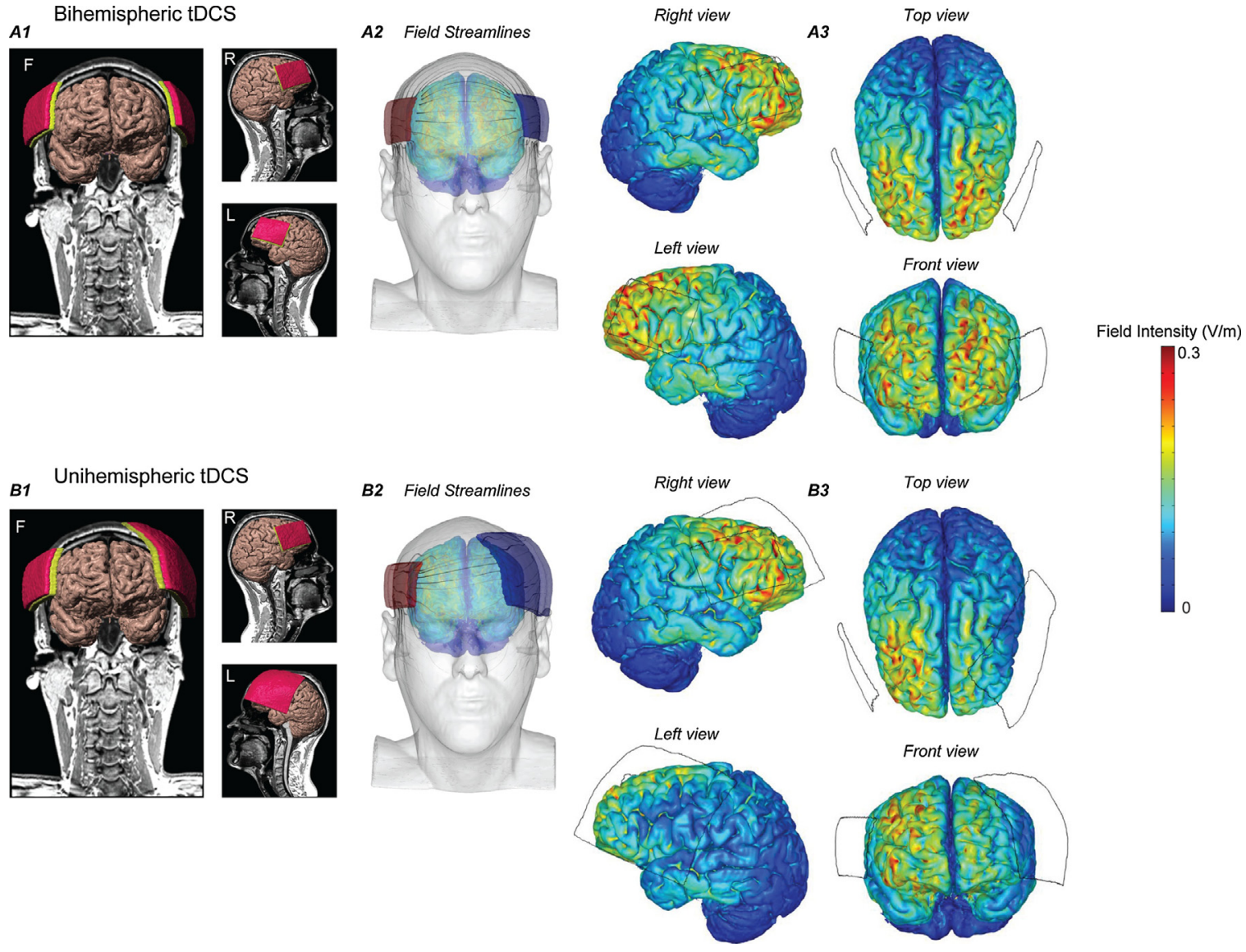

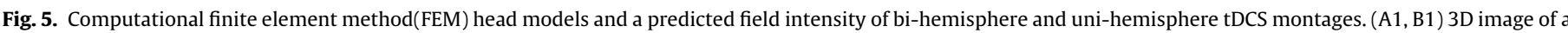

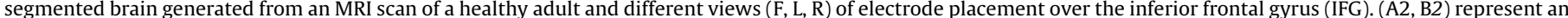

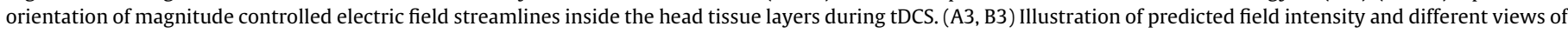

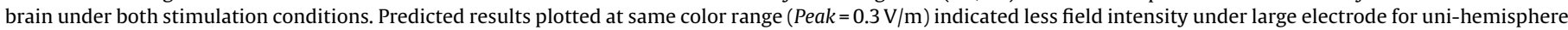
tDCS, whereas for dual-hemisphere tDCS, field intensity was comparable under both anode and cathode.

pare the effects of bilateral vs unilateral anodal tDCS over the IFG, also assessing its impact on the SAT.It is also important to explore alternative explanations: for instance, it is possible that variability during the bihemispheric condition led to the null result. However, it seems that bihemispheric tDCS actually led to a distinct effect from the one induced by unihemispheric tDCS: as it seems that speed increased at the cost of accuracy when compared to sham tDCS. So there is the possibility that with an increased sample size, bihemispheric tDCS would likely seem to increase speed, but at a cost of accuracy. Thus, future studies should explore this difference in order to assess if bihemispheric tDCS was able to actually induce this inverted SAT pattern.

Another important finding is the issue of uni vs. bihemispheric stimulation. Although we showed here that bihemispheric stimulation annuls the beneficial effect of unihemispheric stimulation; results may also be seen as both having a distintic signature: while unihemispheric leads the SAT towards the accuracy tradeoff, bihemispheric may lead towards the opposite result (though here we donit have the statistical power to confirm this result). Regardless, one important recommendation can be derived from the present findings. When using tDCS in cognitive studies, it is important to understand the distinct effects of unihemispheric and bihemispheric tDCS as to find out which one is better. In fact, there are several studies exploring the potential benefits of bihemispheric stimulation. For instance, Meinzer et al. (2014) showed that both single and bihemispheric tDCS over the M1 region significantly improved word-retrieval. Moreover, bihemispheric tDCS when comparing to single hemispheric tDCS seems to activate a widespread network, with stronger bilateral activations when left or right hand were used during a fMRI finger tapping task (Lindenberg et al., 2013). Additionally, bihemispheric montages have been used previously with several effects on decision making, craving reduction, and planning ability, among others (Batista et al., 2015; Fecteau et al., 2007; Leite et al., 2013; Loftus et al., 2015). Thus, it is important to understand the effects of the interaction between the task activated network, alongside with the effects of tDCS over that network. For instance, in order to perform a task, homotopic brain regions can collaborate, or operate independently (segregation) (Davis and Cabeza, 2015). In our study, shifting the balance towards the right hemisphere impaired the effects of tDCS over the right inferior frontal gyrus. Therefore, if one would want to disrupt response control, bihemispheric stimulation may be an appropriate montage.

It is also important to stress that the high definition FEM model is not intended to be used to predict the distribution of the electrical current with gyri-level precision (Bikson et al., 2012; Datta et al., 2009; Ruffini et al., 2014). Instead, the model is used to understand current distribution (Faria et al., 2011; Miranda et al., 2009; Miranda et al., 2006), namely a symmetrical field intensity was predicted for the dual-hemisphere tDCS montage, whereas, field prediction was asymmetrical (smaller field intensity/current density under large electrode compared to the small electrode) for the uni-hemisphere tDCS montage. These predictions are aligned with our initial hypothesis, and thus support the use of such montages to test the effects of dual vs uni-hemispheric tDCS. 
Finally, we did not find any inhibitory effects of tDCS over the IFG on the go/no go task. This suggests that the effects of unihemispheric tDCS on proactive control were task specific. This specificity of tDCS has been shown extensively in the literature, and is thought to be dependent on the activated network, the extension of its activation, its resting state, or the level of cognitive demands (Carvalho et al., 2015; Ehlis et al., 2016; Gill et al., 2015; Hsu et al., 2016).

In sum, this study shows that the effects of unihemispheric tDCS of the right inferior frontal gyrus resemble a pattern of speed accuracy tradeoff (SAT), in which increased task performance accuracy is associated with decreased response speed. As the results disappeared when right IFG was combined with left cathodal IFG tDCS, it is possible that the left IFG is also a critical area in the control of response inhibition and needs to be further considered in the network controlling this behavior.

\section{Conflict of interests}

MB is affiliated with CUNY. CUNY has patents on brain stimulation with MB as inventor. MB has equity in Soterix Medical Inc. The other authors report no conflicts of interest.

\section{Acknowledgements}

JL, SC, OFG are members of CIPSi, supported by the Portuguese Foundation for Science and Technology and the Portuguese Ministry of Science, Technology and Higher Education through national funds and co-financed by FEDER through COMPETE2020 under the PT2020 Partnership Agreement (POCI-01-0145-FEDER-007653); and also through the Portuguese Foundation for Science and Technology PTDC/MHC-PCN/3950/2014. FF is funded by the following NIH grants: R21HD079048, R01HD082302, 1R44NS080632-01, 1R44AT008637, HD069776. SC is funded by the following Portuguese Fondation for Science and Technology (FCT) grant IF/00091/2015.

\section{References}

Aron, A.R., Poldrack, R.A., 2006. Cortical and subcortical contributions to Stop signal response inhibition: role of the subthalamic nucleus. J. Neurosci. 26, 2424-2433.

Aron, A.R., Robbins, T.W., Poldrack, R.A., 2004. Inhibition and the right inferior frontal cortex. Trends Cogn. Sci. 8, 170-177.

Aron, A.R., Durston, S., Eagle, D.M., Logan, G.D., Stinear, C.M., Stuphorn, V., 2007. Converging evidence for a fronto-basal-ganglia network for inhibitory control of action and cognition. J. Neurosci. 27, 11860-11864.

Aron, A.R., Robbins, T.W., Poldrack, R.A., 2014. Right inferior frontal cortex: addressing the rebuttals. Front. Hum. Neurosci. 8, 905.

Bari, A., Robbins, T.W., 2013. Inhibition and impulsivity: behavioral and neural basis of response control. Prog. Neurobiol. 108, 44-79.

Batista, E.K., Klauss, J., Fregni, F., Nitsche, M.A., Nakamura-Palacios, E.M., 2015. A Randomized Placebo-Controlled Trial of Targeted Prefrontal Cortex Modulation with Bilateral tDCS in Patients with Crack-Cocaine Dependence. Int. J. Neuropsychopharmacol. 18 .

Bikson, M., Rahman, A., Datta, A., 2012. Computational models of transcranial direct current stimulation. Clin. EEG Neurosci. 43, 176-183.

Bikson, M., Truong, D.Q., Mourdoukoutas, A.P., Aboseria, M., Khadka, N., Adair, D., Rahman, A., 2015. Modeling sequence and quasi-uniform assumption in computational neurostimulation. Prog. Brain Res. 222, 1-23.

Bogacz, R., Wagenmakers, E.J., Forstmann, B.U., Nieuwenhuis, S., 2010. The neural basis of the speed-accuracy tradeoff. Trends Neurosci. 33, 10-16.

Bolognini, N., Fregni, F., Casati, C., Olgiati, E., Vallar, G., 2010. Brain polarization of parietal cortex augments training-induced improvement of visual exploratory and attentional skills. Brain Res. 1349, 76-89.

Carvalho, S., Boggio, P.S., Goncalves, O.F., Vigario, A.R., Faria, M., Silva, S., Gaudencio do Rego, G., Fregni, F., Leite, J., 2015. Transcranial direct current stimulation based metaplasticity protocols in working memory. Brain Stimul. 8, 289-294.

Cunillera, T., Fuentemilla, L., Brignani, D., Cucurell, D., Miniussi, C., 2014. A simultaneous modulation of reactive and proactive inhibition processes by anodal tDCS on the right inferior frontal cortex. PLoS One 9.

Davis, S.W., Cabeza, R., 2015. Cross-hemispheric collaboration and segregation associated with task difficulty as revealed by structural and functional connectivity. J. Neurosci. 35, 8191-8200.
Davis, S.W., Kragel, J.E., Madden, D.J., Cabeza, R., 2012. The architecture of crosshemispheric communication in the aging brain: linking behavior to functional and structural connectivity. Cereb. Cortex 22, 232-242.

Ditye, T., Jacobson, L., Walsh, V., Lavidor, M., 2012. Modulating behavioral inhibition by tDCS combined with cognitive training. Exp. Brain Res. 219, 363-368.

Ehlis, A.C., Haeussinger, F.B., Gastel, A., Fallgatter, A.J., Plewnia, C., 2016. Taskdependent and polarity-specific effects of prefrontal transcranial direct current stimulation on cortical activation during word fluency. Neuroimage 140 $134-140$.

Erika-Florence, M., Leech, R., Hampshire, A., 2014. A functional network perspective on response inhibition and attentional control. Nat. Commun. 5, 4073.

Faria, P., Hallett, M., Miranda, P.C., 2011. A finite element analysis of the effect of electrode area and inter-electrode distance on the spatial distribution of the current density in tDCS. J. Neural. Eng. 8.

Fecteau, S., Pascual-Leone, A., Zald, D.H., Liguori, P., Theoret, H., Boggio, P.S., Fregni, F., 2007. Activation of prefrontal cortex by transcranial direct current stimulation reduces appetite for risk during ambiguous decision making. J. Neurosci. 27, 6212-6218.

Frank, M.J., Scheres, A., Sherman, S.J., 2007. Understanding decision-making deficits in neurological conditions: insights from models of natural action selection. Philos. Trans. R Soc. Lond. B Biol. Sci. 362, 1641-1654.

Fregni, F., Boggio, P.S., Nitsche, M., Bermpohl, F., Antal, A., Feredoes, E., Marcolin, M.A. Rigonatti, S.P., Silva, M.T., Paulus, W., Pascual-Leone, A., 2005. Anodal transcranial direct current stimulation of prefrontal cortex enhances working memory. Exp. Brain Res. 166, 23-30.

Garavan, H., Ross, T.J., Stein, E.A., 1999. Right hemispheric dominance of inhibitory control: an event-related functional MRI study. Proc. Natl. Acad. Sci. U. S. A. 96, $8301-8306$

Garavan, H., Hester, R., Murphy, K., Fassbender, C., Kelly, C., 2006. Individual differences in the functional neuroanatomy of inhibitory control. Brain Res. 1105, $130-142$.

Gill, J., Shah-Basak, P.P., Hamilton, R., 2015. It's the thought that counts: examining the task-dependent effects of transcranial direct current stimulation on executive function. Brain Stimul. 8, 253-259.

Hampshire, A., Chamberlain, S.R., Monti, M.M., Duncan, J., Owen, A.M., 2010. The role of the right inferior frontal gyrus: inhibition and attentional control. Neuroimage 50, 1313-1319.

Hogeveen, J., Grafman, J., Aboseria, M., David, A., Bikson, M., Hauner, K.K., 2016. Effects of high-Definition and conventional tDCS on response inhibition. Brain Stimul. 9, 720-729.

Hsu, T.Y., Juan, C.H., Tseng, P., 2016. Individual differences and state-Dependent responses in transcranial direct current stimulation. Front. Hum. Neurosci. 10, 643.

Jacobson, L., Javitt, D.C., Lavidor, M., 2011. Activation of inhibition: diminishing impulsive behavior by direct current stimulation over the inferior frontal gyrus. J. Cogn. Neurosci. 23, 3380-3387.

Jacobson, L., Koslowsky, M., Lavidor, M., 2012. tDCS polarity effects in motor and cognitive domains: a meta-analytical review. Exp. Brain Res. 216, 1-10.

Kelly, A.M., Hester, R., Murphy, K., Javitt, D.C., Foxe, J.J., Garavan, H., 2004. Prefrontal-subcortical dissociations underlying inhibitory control revealed by event-related fMRI. Eur. J. Neurosci. 19, 3105-3112.

Kim, D., Joo, E.Y., Tae, W.S., Han, S.J., Cho, J.W., Seo, D.W., Hong, S.B., 2007. Cortical localization of scalp electrodes on three-dimensional brain surface using frameless stereotactic image guidance system. J. Korean Neurol. Assoc. 25, 155-160.

Kunze, T., Hunold, A., Haueisen, J., Jirsa, V., Spiegler, A., 2016. Transcranial direct current stimulation changes resting state functional connectivity: a large-scale brain network modeling study. Neuroimage.

Leite, J., Carvalho, S., Fregni, F., Goncalves, O.F., 2011. Task-specific effects of tDCSinduced cortical excitability changes on cognitive and motor sequence set shifting performance. PLoS One 6.

Leite, J., Carvalho, S., Fregni, F., Boggio, P.S., Goncalves, O.F., 2013. The effects of cross-hemispheric dorsolateral prefrontal cortex transcranial direct current stimulation (tDCS) on task switching. Brain Stimul. 6, 660-667.

Loftus, A.M., Yalcin, O., Baughman, F.D., Vanman, E.J., Hagger, M.S., 2015. The impact of transcranial direct current stimulation on inhibitory control in young adults. Brain Behav. 5.

Meinzer, M., Lindenberg, R., Sieg, M.M., Nachtigall, L., Ulm, L., Flöel, A., 2014. Transcranial direct current stimulation of the primary motor cortex improves word-retrieval in older adults. Front. Aging Neurosci. 6, 253.

Miranda, P.C., Lomarev, M., Hallett, M., 2006. Modeling the current distribution during transcranial direct current stimulation. Clin. Neurophysiol. 117, 1623-1629.

Mondino, M., Jardri, R., Suaud-Chagny, M.F., Saoud, M., Poulet, E., Brunelin, J., 2016. Effects of fronto-Temporal transcranial direct current stimulation on auditory verbal hallucinations and resting-State functional connectivity of the left temporo-Parietal junction in patients with schizophrenia. Schizophr. Bull. 42, 318-326.

Morales-Quezada, L., Leite, J., Carvalho, S., Castillo-Saavedra, L., Cosmo, C., Fregni, F., 2016. Behavioral effects of transcranial pulsed current stimulation (tPCS): Speed-accuracy tradeoff in attention switching task. Neurosci. Res. 109, 48-53.

Nitsche, M.A., Paulus, W., 2000. Excitability changes induced in the human motor cortex by weak transcranial direct current stimulation. J. Physiol. 527 (Pt 3), 633-639.

Nitsche, M.A., Paulus, W., 2001. Sustained excitability elevations induced by transcranial DC motor cortex stimulation in humans. Neurology 57, 1899-1901. 
Nitsche, M.A., Doemkes, S., Karakose, T., Antal, A., Liebetanz, D., Lang, N., Tergau, F., Paulus, W., 2007. Shaping the effects of transcranial direct current stimulation of the human motor cortex. J. Neurophysiol. 97, 3109-3117.

Peterchev, A.V., Wagner, T.A., Miranda, P.C., Nitsche, M.A., Paulus, W., Lisanby, S.H. Pascual-Leone, A., Bikson, M., 2012. Fundamentals of transcranial electric and magnetic stimulation dose: definition, selection, and reporting practices. Brain Stimul. 5, 435-453.

Philipp, A.M., Weidner, R., Koch, I., Fink, G.R., 2013. Differential roles of inferior frontal and inferior parietal cortex in task switching: evidence from stimuluscategorization switching and response-modality switching. Hum. Brain Mapp. 34, 1910-1920.

Ruffini, G., Fox, M.D., Ripolles, O., Miranda, P.C., Pascual-Leone, A., 2014. Optimization of multifocal transcranial current stimulation for weighted cortical pattern targeting from realistic modeling of electric fields. Neuroimage 89, 216-225.

Snitz, B.E., MacDonald 3rd, A., Cohen, J.D., Cho, R.Y., Becker, T., Carter, C.S., 2005. Lateral and medial hypofrontality in first-episode schizophrenia: functional activity in a medication-naive state and effects of short-term atypical antipsychotic treatment. Am. J. Psychiatry 162, 2322-2329.

Stramaccia, D.F., Penolazzi, B., Sartori, G., Braga, M., Mondini, S., Galfano, G., 2015. Assessing the effects of tDCS over a delayed response inhibition task by targeting the right inferior frontal gyrus and right dorsolateral prefrontal cortex. Exp. Brain Res. 233, 2283-2290.
Swick, D., Ashley, V., Turken, U., 2008. Left inferior frontal gyrus is critical for response inhibition. BMC Neurosci. 9, 102

Wietschorke, K., Lippold, J., Jacob, C., Polak, T., Herrmann, M.J., 2016. Transcranial direct current stimulation of the prefrontal cortex reduces cue-reactivity in alcohol-dependent patients. J. Neural. Transm (Vienna).

Witt, S.T., Stevens, M.C., 2013. The role of top-down control in different phases of a sensorimotor timing task: a DCM study of adults and adolescents. Brain Imaging Behav. 7, 260-273.

Yavari, F., Shahbabaie, A., Leite, J., Carvalho, S., Ekhtiari, H., Fregni, F., 2016. Noninvasive brain stimulation for addiction medicine: from monitoring to modulation. Prog. Brain Res. 224, 371-399.

Zaehle, T., Sandmann, P., Thorne, J.D., Jancke, L., Herrmann, C.S., 2011. Transcranial direct current stimulation of the prefrontal cortex modulates working memory performance: combined behavioural and electrophysiological evidence. BMC Neurosci. 12, 2

van Rooij, S.J., Rademaker, A.R., Kennis, M., Vink, M., Kahn, R.S., Geuze, E., 2014 Impaired right inferior frontal gyrus response to contextual cues in male veterans with PTSD during response inhibition. J. Psychiatry Neurosci. 39, 330-338. 